\title{
Design and Control of Femtosecond Lasers for Optical Clocks and the Synthesis of Low-Noise Optical and Microwave Signals
}

\author{
Scott A. Diddams, Albrecht Bartels, Tanya M. Ramond, Chris W. Oates, S. Bize, E. A. Curtis, J. C. Bergquist, \\ and Leo Hollberg
}

\begin{abstract}
This paper describes recent advances in the design and control of femtosecond laser combs for their use in optical clocks and in the synthesis of low-noise microwave and optical signals. The authors present a compact and technically simple femtosecond laser that directly emits a broad continuum and shows that it can operate continuously on the timescale of days as the phase-coherent "clockwork" of an optical clock. They further demonstrate phase locking of an octave-spanning frequency comb to an optical frequency standard at the millihertz level. As verified through heterodyne measurements with an independent optical frequency standard, this provides a network of narrow optical modes with linewidths at the level of $\leq 150 \mathrm{~Hz}$, presently limited by measurement noise. Finally, they summarize their progress in using the femtosecond laser comb to transfer the stability and low phase-noise optical oscillators to the microwave domain.
\end{abstract}

Index Terms-Frequency metrology, frequency synthesis, optical clocks, ultrafast optics and lasers.

\section{INTRODUCTION}

$\mathbf{S}^{\mathrm{n}}$ INCE femtosecond lasers were introduced into the field of optical frequency metrology about four years ago [1], [2], they have become indispensable tools in this exciting and expanding area of research [3]-[5]. It is now widely accepted that mode-locked femtosecond lasers will play a critical role in the next generation of atomic clocks based on optical frequencies [6], [7]. In this role, the femtosecond laser (sometimes in conjunction with nonlinear optical fiber) serves as the "optical clockwork" or "synthesizer" that phase-coherently divides the uncountable rate of optical cycles to a countable microwave frequency for subsequent use and comparison to existing standards. The connection between optical and microwave domains is understood most readily in the frequency domain, where the spectrum of the femtosecond laser consists of a comb of evenly spaced modes with frequencies given by

$$
f_{n}=n f_{r}+f_{o} .
$$

Here, $f_{r}$ is the repetition frequency of the laser (typically 0.1 to $1 \mathrm{GHz}$ ), $n$ is an integer, and $f_{o}$ is a common offset

Manuscript received January 17, 2003.

S. A. Diddams, A. Bartels, T. M. Ramond, C. W. Oates, E. A. Curtis, J. C. Bergquist, and L. Hollberg are with the Time and Frequency Division, National Institute of Standards and Technology (NIST), Boulder, CO 80305 USA.

S. Bize is with the Time and Frequency Division, National Institute of Standards and Technology (NIST), Boulder, CO 80305 USA. He is also with BNM-SYRTE, Observatoire de Paris, 75014 Paris, France (e-mail: sdiddams@boulder.nist.gov).

Digital Object Identifier 10.1109/JSTQE.2003.819096 frequency-sometimes called the carrier-envelope offset frequency - due to dispersion in the laser cavity. We have experimentally tested the validity of (1) [8], as have others [2], [9]-[11], to uncertainties approaching one part in $10^{18}$.

With these facts established, we have now begun to turn our attention toward the more practical issue of actually making a robust and reliable optical clockwork that functions in a manner more akin to radio-frequency and microwave synthesizers (e.g., a turnkey device that could readily operate for days and weeks). In spite of its very desirable properties, the nonlinear microstructure fiber used in conjunction with the femtosecond laser is often found to be the weak point when we consider the reliability of present optical clockworks. The recent introduction of lasers that emit octave-spanning spectra directly, and thereby circumvent the need for nonlinear spectral broadening in microstructure fibers, is an important advance toward a more reliable clockwork. Section II of this paper describes our efforts in this direction. We present details of a technically elegant broad-band femtosecond laser with 1-GHz repetition rate and its use as an optical clockwork that does not employ a microstructure optical fiber. As will be shown, this system can be tightly phase locked to an optical oscillator for periods approaching 1 day. With improved thermal control, we expect this could be extended to indefinite periods.

On a second front, we continue to explore new opportunities that arise as our control of the femtosecond laser improves. Specifically, while our earlier work has demonstrated that the associated mode comb of the optical clockwork can be exceedingly stable [8], we now show that we are able to make the linewidth of the elements of the mode comb reproduce that of an optical frequency standard at a level that begins to be interesting $(\leq 150 \mathrm{~Hz})$. Tight control of the octave-spanning optical comb means that we can now envision the phase-coherent transfer of not only the stability, but also the linewidth of a very narrow optical oscillator to several hundred thousand comb elements spanning the visible and near infrared spectrum. Such an array of narrow optical oscillators would be a valuable general tool for spectroscopy, and the tight phase control will also be critical for the creation of low-noise microwave signals that are generated by dividing down optical oscillators with the femtosecond optical clockwork. It seems clear that in the near future the ultimate stability and phase noise performance from any electromagnetic oscillator will belong to a laser referenced to narrow atomic transitions. The challenging task of using the femtosecond laser to transfer the properties of optical oscillators across the optical 


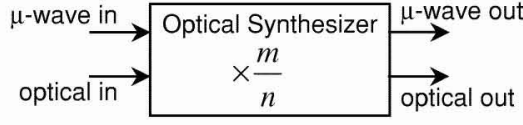

Fig. 1. Broad-band frequency comb of femtosecond laser can be viewed as a general purpose optical frequency synthesizer. It can use either a microwave ( $\mu$-wave) or optical input reference that is phase-coherently linked to an optical or microwave frequency at the output.

spectrum and to the microwave domain is presented in Section III.

\section{GENERATION AND CONTROL OF OCTAVE-SPANNING Spectra Directly From a Femtosecond Laser}

A femtosecond laser and its associated frequency comb can be viewed as a general purpose extremely broad-band synthesizer with either an optical or microwave frequency reference (Fig. 1). As will be described below, the output of the synthesizer can similarly be an optical or microwave frequency. To accomplish this, it is required that both degrees of freedom of the frequency comb be measured and controlled. Whereas this task is easily solved for the repetition rate $f_{r}$ by monitoring the pulse train with a sufficiently fast photodetector, it is more challenging to measure the offset frequency $f_{0}$ of the comb. In the most common scheme it is required that the laser is spectrally broadened to cover more than one octave. Then one can employ a so-called self-referencing technique [12] in which low-frequency components of the fundamental spectrum are frequency doubled and heterodyned with a spectrally matching high-frequency slice to generate a beatnote at frequency $f_{o}$. Starting with the typical $\sim 20 \mathrm{~nm}$ bandwidth of a Ti:sapphire laser, spectral broadening to more than an octave is easily accomplished through self-phase-modulation in microstructured fibers [13]. While these fibers have performed amazingly well in all experiments to date, they can present challenging technical limitations on the ability to control the femtosecond laser over long periods. Coupling of the light into their $\sim 1.7 \mu \mathrm{m}$ cores is difficult to maintain for long times at a level where an octave of spectrum is attained. Also, even in relatively clean labs we have found that slow degradation of the fiber faces can occur, especially when subject to high average powers. Because of the high nonlinearity employed, small fluctuations and drifts in the coupling strongly modify the output spectrum and thereby decrease the signal-to-noise ratio (SNR) in the subsequent optical beats. In our experience, this typically limits reliable (hands-off) access to and control of $f_{o}$ to a few hours. Beyond these technical limitations, Corwin, et al. have also identified and studied noise of a more fundamental nature (shot noise) that can be amplified to exceedingly high levels in microstructured fibers and, thereby, limit its utility [14].

\section{A. Broad-Band Continuum Generation From a Ti:Sapphire Laser}

The development of femtosecond lasers that span a broad-band continuum directly has circumvented the aforementioned problems with spectral broadening in fibers. Two practical routes toward shorter pulses and broader spectra in a femtosecond oscillator have been used. One approach is to engineer the group-delay dispersion (GDD) inside a resonator

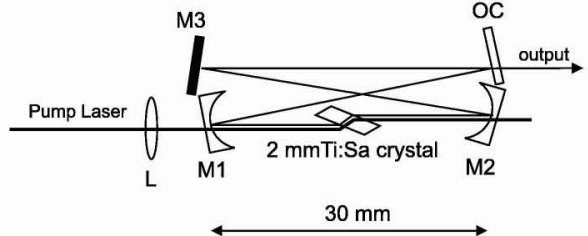

Fig. 2. Cavity of the broad-band femtosecond laser. Pump laser is focused through lens $L$ (focal length $f=30 \mathrm{~mm}$ ) into the 2-mm-long Ti:sapphire crystal. Cavity is formed by the two concave mirrors M1 and M2 $(f=15 \mathrm{~mm})$, the convex mirror M3 $(f=-0.5 \mathrm{~m})$, and the output coupler OC. Unidirectional output is indicated.

in a way that short pulses with extremely broad spectra do not spread significantly in time during one cavity round trip. This involves careful design of dispersive mirrors that have negative GDD over the desired large bandwidth with low higher order dispersion. Alternatively, one can allow higher order dispersion in the resonator, but at the same time ensure that the leading and trailing edges of the temporally spreading pulse are sufficiently suppressed, such that only a short pulse remains stable in the cavity. This can be achieved by an effective saturable absorber or an instantaneous power-dependent gain, where both effects are more generally called self-amplitude modulation (SAM). The first approach has been taken with great success by Ell et al. [15]. Their carefully dispersion-engineered resonator is capable of generating spectra exceeding one octave with 5-fs pulses at a $64-\mathrm{MHz}$ repetition rate. With this laser it has been possible to measure $f_{o}$ in a self-referencing scheme without spectral broadening in fibers [16]. In contrast to that approach, we have pursued the second route of employing stronger SAM in a standard laser while the higher order dispersion characteristics remained unchanged. We modified a ring oscillator that has been used in the past to generate relatively narrow-band $(\sim 30 \mathrm{~nm})$ spectra [17].

The ring cavity of our 1-GHz repetition rate laser is sketched in Fig. 2. A 2-mm-long Ti:sapphire crystal is placed at Brewster's angle between two concave mirrors M1 and M2 with radius of curvature of $30 \mathrm{~mm}$. It is pumped through lens $L$ (focal length $30 \mathrm{~mm}$ ) by $8.5 \mathrm{~W}$ from a frequency-doubled $\mathrm{Nd}: \mathrm{YVO}_{4}$ laser. The resonator is completed by mirror M3 and the output coupler OC. Mirrors M1-M3 have a negative dispersive coating with a relatively narrow-band operating range from 750 to 850 $\mathrm{nm}$ and nonnegligible higher order dispersion outside this band. As has been described by Bartels et al. [18], replacing the usual flat mirror at position M3 with a convex mirror having a radius of curvature of $1 \mathrm{~m}$ leads to an increase of the self-induced gain-modulation through the soft-aperture Kerr-lens effect. This presents stronger SAM in the cavity and acts to efficiently "clean up" the pulse spreading inside the resonator that is caused by higher order dispersion. Extraordinarily broad continuum emission has been achieved, and a typical unidirectionally mode-locked spectrum is shown in Fig. 3. It ranges from approximately 630 to $945 \mathrm{~nm}$ at $10 \mathrm{~dB}$ below the maximum. The corresponding average output power is $850 \mathrm{~mW}$.

\section{B. Linking the Broad-Band Femtosecond Laser to an Optical Reference Oscillator}

Compared to the earlier mentioned device presented by Ell et al. [15], our laser has a lower peak power due to its much higher 


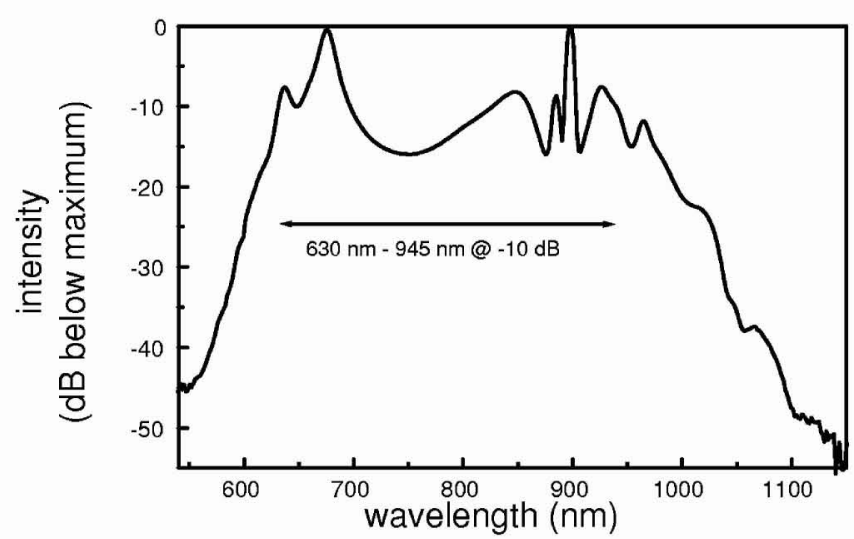

Fig. 3. Spectrum of the broad-band laser. It ranges from approximately 630 to $945 \mathrm{~nm}$ at $-10 \mathrm{~dB}$ below its maximum. Total output power is $850 \mathrm{~mW}$.

repetition rate, which makes the necessary nonlinear spectral broadening less effective. Therefore, the low power in the spectral wings of our spectrum is insufficient to employ the conventional self-referencing scheme of beating the second harmonic of an infrared part of the spectrum against a fundamental visible part. We need to take a similar but slightly more complicated route that was first realized by Morgner et al. [16] to measure $f_{o}$ without broadening in a fiber. The idea is to frequency double a spectral slice around the $622-\mathrm{nm}$ wavelength, leading to frequencies $f_{m}=2 f_{o}+2 m f_{r}$, and to frequency triple a spectral slice around $933 \mathrm{~nm}$, leading to frequencies $f_{n}=3 f_{o}+3 n f_{r}$, both around $311 \mathrm{~nm}$. Under these conditions $m=3 n / 2$, and a heterodyne beat between the ultraviolet (UV) beams then gives $f_{o}$.

The experimental setup to measure $f_{o}$ of our laser is shown in Fig. 4. A beamsplitter (BS1) reflects the spectrum from approximately 600 to $740 \mathrm{~nm}$ into the second harmonic generation (SHG) arm of our nonlinear interferometer. The remainder of the spectrum is transmitted through BS1 and enters the third-harmonic generation (THG) arm. In the SHG arm a 0.3 -mm-thick type-I phase-matched $\beta$-barium-borate (BBO) crystal is angle-tuned to produce SHG light around 311 $\mathrm{nm}$. In the THG arm, light is frequency tripled in two steps to also produce $311-\mathrm{nm}$ wavelength. We first frequency double the infrared light in an angle-tuned 2-mm-thick $\mathrm{KNbO}_{3}$ crystal to produce $\approx 465 \mathrm{~nm}$. Then, the infrared and blue portions are separated with beamsplitter BS2. The $465-\mathrm{nm}$ light passes through a halfwave plate while the infrared part passes a delay stage before both beams are recombined and focused into a 0.5-mm-thick BBO crystal for type-I sum-frequency generation (SFG) of light at $311 \mathrm{~nm}$. The $311-\mathrm{nm}$ beams from both arms are subsequently superimposed on the $50 \%$ beamsplitter BS4.

To generate a beat signal between the two UV beams with optimized SNR, several essential requirements need to be fulfilled. First, in the THG arm, the blue and infrared pulses, which, in general, are not temporally coincident due to dispersion, need to be overlapped in order to achieve SFG. The required accuracy in the setting of the delay stage used for this purpose is approximately $20 \mu \mathrm{m}$. Second, the UV light coming from both arms needs to be monitored with a UV spectrometer to ensure that they spectrally overlap with an accuracy of approximately $1 \mathrm{~nm}$. Third, at the same time, the angle of the nonlinear crystal in the SHG arm needs to be tuned so that the amount of SHG light

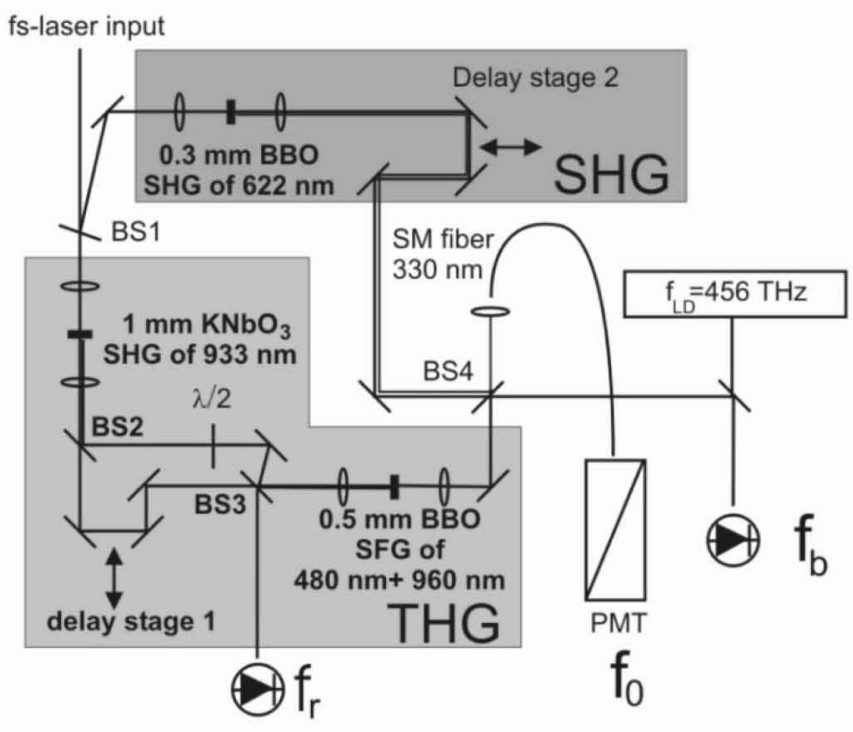

Fig. 4. Setup for the measurement of $f_{o}$.

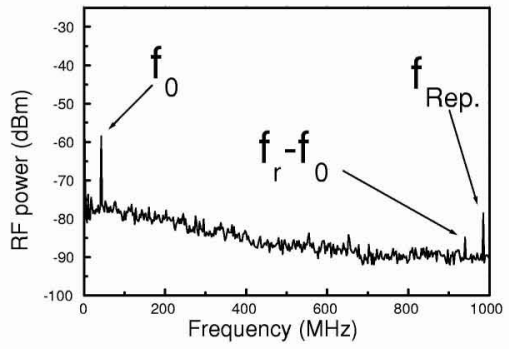

Fig. 5. Radio-frequency spectrum of the beatnote between the SHG and THG portions of the comb, showing $f_{o}$, its image $f_{r}-f_{o}$, and the repetition rate. Data were taken with a resolution bandwidth of $300 \mathrm{kHz}$.

that is not spectrally overlapped with the THG is minimized. Nonspectrally matched components only add to the detected noise level. Fourth, the two UV beams must be mode matched. This requirement is difficult to achieve in free space as both UV beams have travelled through several (nonlinear) optical components and thus have beam profiles far from the ideal Gaussian. Therefore, we use a single-mode fiber for $330 \mathrm{~nm}$ to spatially filter both beams before detection of the beatnote with a photomultiplier tube (PMT). And finally, the two UV pulses also must temporally overlap. This ensures that, in fact, the many heterodyne beats between adjacent components of the secondand third-harmonic frequency combs add up phase coherently. An example of such a beat signal is shown in Fig. 5, where we present the radio-frequency spectrum of the PMT signal over the range from $0 \mathrm{~Hz}$ to the $1-\mathrm{GHz}$ repetition rate. The signal at $f_{o}$ as well as its image $f_{r}-f_{o}$ are visible. The $f_{o}$ signal usually has a SNR of $22 \mathrm{~dB}$ in a $300-\mathrm{kHz}$ bandwidth. The image signal has a lower SNR because of the limited PMT bandwidth.

Having access to both degrees of freedom of our frequency comb, we are able to phase-coherently link it to a microwave or optical reference oscillator of our choice [19]. Here, we choose the single-frequency light from a laser diode that has its frequency $f_{\mathrm{LD}} \approx 456 \mathrm{THz}(657 \mathrm{~nm})$ locked to a high-finesse Fabry-Pérot cavity. This stabilized laser diode is the reference oscillator for the NIST calcium optical standard, so its frequency can readily be locked to the narrow ${ }^{1} S_{0}\left(m_{j}=0\right) \leftrightarrow{ }^{3} P_{1}\left(m_{j}=0\right)$ 

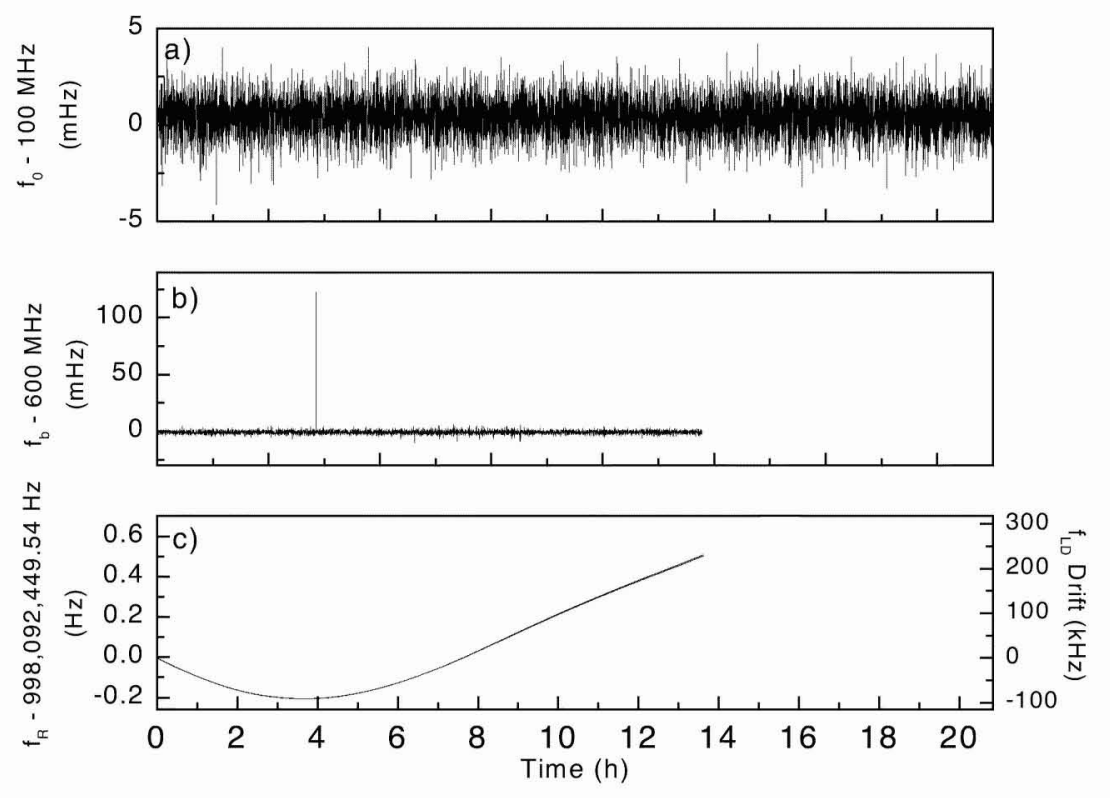

Fig. 6. Record of simultaneous frequency counter readings at gate time of $10 \mathrm{~s}$ of (a) $f_{o}$, (b) $f_{b}$, and (c) the repetition rate $f_{r}$ of the phase-locked laser. Right axis in (c) is equivalent to the left axis multiplied by $n_{0}$ (the index of the mode that has been locked to $f_{\mathrm{LD}}$ ) and allows inference of the temporal drift of $f_{\mathrm{LD}}$.

clock transition in an ensemble of laser-cooled neutral ${ }^{40} \mathrm{Ca}$ atoms [20], [21]. To link the comb to $f_{\mathrm{LD}}, f_{o}$ is phase locked to a synthesizer that is referenced to a hydrogen maser by acting on the pump power via an acousto-optic modulator (AOM). Additionally, a heterodyne beat signal between $f_{\mathrm{LD}}$ and the neighboring comb component with mode element $n_{0} \approx 456000$ is detected at frequency $f_{b}$. For this purpose, we overlap the beam passing through BS4 with the light from the laser diode on a photodiode (see Fig. 4). $f_{b}$ is phase locked to a synthesizer by acting on the cavity length of the femtosecond laser through a mirror that is mounted onto a piezo-electric transducer (PZT). The result is that the repetition rate is phase coherently linked to $f_{\mathrm{LD}}$ as

$$
f_{r}=\frac{1}{n_{0}}\left(f_{\mathrm{LD}}-f_{b}-f_{o}\right) .
$$

With both degrees of freedom of the comb thus phase locked, $f_{r}$ is monitored by focusing onto a photodiode the infrared light that is reflected off of BS3. Furthermore, if we redefine the origin of the comb, we can now rewrite (1) as

$$
f_{k}=f_{\mathrm{LD}}-f_{b} \pm \frac{k}{n_{0}}\left(f_{\mathrm{LD}}-f_{o}-f_{b}\right)
$$

with $k$ being an integer (including zero). In this expression, we see the effective origin of our frequency comb is $f_{\mathrm{LD}}-f_{b}$ and the mode-spacing is, as always, given by the repetition rate (2). Assuming that we stabilize both $f_{o}$ and $f_{b}$ to $0 \mathrm{~Hz}$, which in principle is always possible, it becomes apparent that indeed $f_{r}$ as well as all optical frequencies $f_{k}$ of the comb are phase locked to $f_{\mathrm{LD}}$. More generally, it can be shown that even if $f_{o}$ and $f_{b}$ have nonzero frequencies and the synthesizers used for their phase locks are referenced to an independent microwave standard (such as a hydrogen maser), both the accuracy and the stability of $f_{r}$ and the $f_{k}$ are determined entirely by the optical oscillator $f_{\mathrm{LD}} \cdot{ }^{1}$ In the case where $f_{\mathrm{LD}}$ is locked to the clock transition of the Ca frequency standard, an optical atomic clock with a microwave output is created, where the latter is the laser repetition rate [6]. The phase noise and stability of $f_{r}$ and the $f_{k}$ will be addressed in Section II-C.

\section{Long-Term Operation of the Optical Clockwork}

The phase-coherent link of the frequency comb and its repetition rate to $f_{\mathrm{LD}}$ is monitored by simultaneously counting $f_{o}$, $f_{b}$, and $f_{r}$ with frequency counters operating at 10-s gate time $\tau$. The offsets of the counter readings from their phase-locked values are displayed in Fig. 6 for all three channels. (The phaselocked values are $f_{o}=100 \mathrm{MHz}$ and $f_{b}=600 \mathrm{MHz}$, both defined by the reference frequency used for the phase-locked loop; $f_{r}=998092449.54 \mathrm{~Hz}$, defined by $f_{\mathrm{LD}}$ and the choices of $f_{o}$ and $f_{b}$ ). The femtosecond laser usually maintains mode-locked operation with power fluctuations below $1 \%$ for as long as the pump laser is turned on without noticeable changes to the output spectrum. As a result, the SNR of the $f_{o}$ signal is constant and can be phase locked for arbitrary times. Fig. 6(a) shows cycle-slip-free operation for $21 \mathrm{~h}$ until the pump laser was turned off. Our ability to phase lock $f_{b}$ for long times depends on whether we are able to compensate for the thermal expansion of the laser baseplate with the PZT operation range, presently limited to $1 \mu \mathrm{m}$. To improve this ability, we employ an active temperature control that keeps the laser baseplate at a constant temperature slightly above room temperature. As shown in Fig. 6(b), the phase lock on $f_{b}$ operated for $\approx 14 \mathrm{~h}$ with only one cycle-slip at $\approx 4 \mathrm{~h}$, i.e., $\approx 10 \mathrm{~h}$ of cycle-slip free data are recorded. The reason for the failure at $\approx 14 \mathrm{~h}$ is not clear, although it is likely that the stabilization of $f_{\mathrm{LD}}$ to the Fabry-Pérot cavity failed and prevented our feedback loop from tracking the diode laser frequency. As $f_{r}$ represents a measurement of $f_{\mathrm{LD}}$,

\footnotetext{
${ }^{1}$ This statement is true as long as the absolute frequency uncertainty and stability (i.e., measured in Hertz) of the microwave standard are small compared to those of the optical reference oscillator.
} 
the time record of the offset of $f_{r}$ from $998092449.54 \mathrm{~Hz}$ has been multiplied with the mode number $n_{0}=456857$ to give the temporal drift of the Fabry-Pérot cavity used to stabilize $f_{\mathrm{LD}}$. This is shown on the right axis of the graph in Fig. 6(c). The cycle slip in the $f_{b}$ feedback loop does not appear in the $f_{r}$ record because the effect of the $120-\mathrm{mHz}$ excursion in $f_{b}$ results in an error of $260 \mathrm{nHz}$ in $f_{*}$, which is below our measurement resolution. Evaluating the rms frequency fluctuation of the phase locked $f_{o}$ and $f_{b}$, we find that they impose an instability of $<6 \times 10^{-18}$ in 10 s on the frequency comb, far below the instability of $\int_{\text {LD }}$.

With this, we have demonstrated the ability to count $\sim 1.6 \times$ $10^{19}$ optical cycles at $456-\mathrm{THz}$ frequency without ever losing track of the oscillation. Also, our result implies that we can carry out an optical frequency measurement relative to a microwave frequency standard (in this case the hydrogen maser) with no invalid data points for an uninterrupted period of 14 h. As we move toward the operation of optical standards as clocks, this ability becomes particularly important for measurements where long averaging times are necessary to attain useful data, as well as to test the long term stability limit of optical and microwave frequency standards. The new broad-band laser has thus far shown great potential as an optical-to-microwave conversion tool that operates hands-off for periods approaching one day. This step forward has been made possible by the fact that difficulties with the long-term operation of systems based on microstructure fiber can now be circumvented in an elegant and practical way.

\section{SyNTHESIS OF LOW-NOISE OPTICAL AND MICROWAVE SIGNALS}

The continuous pursuit of optical atomic frequency standards over four decades has been motivated by the promise of impressive stability and the related potential for improved accuracy [22]. These benefits ultimately stem from the use of narrow resonances at very high frequencies. The stability of an atomic frequency standard with frequency $\nu_{0}$ and linewidth $\Delta \nu$ scales as $Q=\nu_{0} / \Delta \nu$, allowing optical standards to be potentially $10^{5}$ times more stable than their microwave counterparts. A necessary requirement for such optical standards is that a suitably stable and spectrally narrow oscillator (i.e., a laser) be used to probe the optical transition. This has led to the development of visible lasers with subhertz linewidths and instabilities as low as $4 \times 10^{-16}$ at $1 \mathrm{~s}$ [23]. As will be discussed in further detail below, such lasers are some of the best oscillators of any kind that exist today.

Given that such stable low-noise optical oscillators presently exist, the problem we now address in the context of this paper is the use of the femtosecond-laser-based frequency comb to transfer the properties of such oscillators across the optical spectrum and to the microwave domain as well. In short, stabilization of the femtosecond frequency comb to an optical standard can potentially be used to transfer the desirable properties of the optical standard to each of the modes of the comb. The various difference frequencies (i.e, $f_{r}$ and its harmonics) between the comb elements should then, in principle, possess the same properties of the optical reference oscillator. Here, it is worth pointing out the difference between our approach and the powerful "transfer oscillator" approach introduced by Telle et al. [24], [25]. In their scheme, a clever choice of frequency mixings effectively eliminates the noise properties of the unstabilized femtosecond laser when it is used for comparing widely separated optical frequencies or even optical and microwave frequencies. The uncertainty of their technique for optical-to-optical comparisons has been verified at the level of $\sim 1 \times 10^{-18}$ [25]. In contrast, the approach we take here is to attempt to tightly stabilize (phase lock) the entire femtosecond comb to the optical standard. The more general end result is the simultaneous creation of half a million stable frequency modes with narrow linewidth that can be used for spectroscopic references and frequency measurements, or even for direct time- or frequency-domain spectroscopy [26], [27]. In earlier work [6], [8], we have shown that the stability of an optical atomic oscillator can be transferred to the modes of a femtosecond comb to the level of $\sim 6 \times 10^{-16}$ in $1 \mathrm{~s}$. However, this same level of transfer instability has not yet been achieved for $\int_{r}$, and the extent to which the narrowness of the optical oscillator is transferred to each of the femtosecond comb teeth has not been explored. In what follows, we will present the current status, the known limitations, and our perspective of the potential that can still be realized in this area.

\section{A. Comparison of Independent Optical Frequency Standards Via the Femtosecond Comb}

In this section, we describe experiments that test our ability to faithfully transfer the narrow frequency spectrum of an optical standard to the elements of the femtosecond laser comb. To accomplish this, we take advantage of the $\mathrm{Hg}^{+}$and $\mathrm{Ca}$ optical standards that exist at NIST. The $\mathrm{Hg}^{+}$standard consists of a well-stabilized 563-nm (532-THz) dye laser whose second harmonic is locked to the center of the $282-\mathrm{nm}$ transition in a single laser-cooled and trapped ${ }^{199} \mathrm{Hg}^{+}$ion [23], [28]. The Ca standard employs a well-stabilized diode laser that has its frequency locked to the $657-\mathrm{nm}(456-\mathrm{THz})$ transition in neutral ${ }^{10} \mathrm{Ca}$ atoms that are laser-cooled and confined in a magnetooptical trap [20], [21]. As discussed above, the femtosecond laser spectrum of Fig. 3 has sufficient power at the $657-\mathrm{nm} \mathrm{Ca}$ color for phase locking the comb; however, at the 563-nm wavelength of the $\mathrm{Hg}^{+}$standard, there is less than $100 \mathrm{pW}$ per mode, which results thus far in a marginal SNR of $10 \mathrm{~dB}(300-\mathrm{kHz}$ bandwidth) for the heterodyne beat. For this reason, we use the 1-GHz microstructure-fiber-based system that has been described in detail in previous publications [8], [28], [29], [30]. While we anticipate that the noise properties of the broad-band laser described in Section II will ultimately be superior, it is not evident that the microstructure fiber imposes any limitation at the current level of measurement precision.

In this experiment, $f_{0}$ of the femtosecond comb created from nonlinear broadening in microstructure fiber [13] is phase locked at $244 \mathrm{MHz}$ to a signal derived from a hydrogen maser using feedback to an AOM in the path of the pump beam. The bandwidth of this loop is limited to $\sim 200 \mathrm{kHz}$ by the transit delay of the acoustic wave in the AOM. One of the comb teeth near $563 \mathrm{~nm}$ is then heterodyned with the dye-laser light of the $\mathrm{Hg}^{+}$standard, which is delivered to the femtosecond laser 


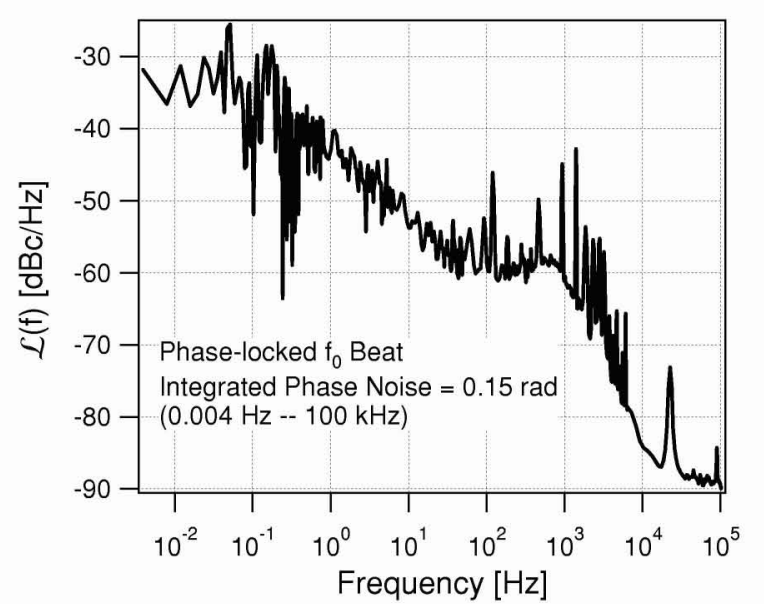

Fig. 7. Spectral density of phase fluctuations of phase-locked offset frequency $f_{0}$.

system by $180 \mathrm{~m}$ of single-mode optical fiber. The resultant beat frequency, $f_{b}$, is then phase locked at $574 \mathrm{MHz}$ to a second maser-derived signal using feedback to a PZT behind a cavity mirror. Here, the loop bandwidth is limited to about $25 \mathrm{kHz}$ by the first mechanical resonance of the PZT/mirror structure. The frequency spectra of these two phase-locked beats are shown in Figs. 7 and 8. Although not directly shown here, the linewidth of $f_{o}$ was measured to be at the millihertz level. To further analyze the quality of the $f_{o}$ phase lock we have used an independent 244-MHz signal and a mixer in the customary manner to translate phase noise into voltage noise that we then record with a high-resolution Fourier-transform spectrum analyzer. The resulting single-side-band phase noise spectrum $\mathcal{L}(f)=10 \log \left[S_{\phi}(f) / 2\right]$ of the phase locked $f_{o}$ beat is displayed in Fig. 7. The integrated phase noise between $0.004 \mathrm{~Hz}$ and $100 \mathrm{kHz}$ is $0.15 \mathrm{rad}$. This very low level is comparable to what was reported in [31] and [32] and indicates that long-term coherence of $f_{o}$ is possible with this high-repetition-rate system.

Although we did not make a similar phase-noise measurement, the spectra shown in Fig. 8 indicate that a tight phase lock (millihertz level) can be accomplished also for $f_{b}$. Fig. 8(a) and (b) shows spectra on two different scales of the phase-locked $f_{b}$ as recorded with a conventional RF spectrum analyzer. In Fig. 8(a), one clearly sees evidence of noise being removed up to the loop bandwidth. The peaks at 113 and $226 \mathrm{kHz}$ are due to amplitude noise on the pump laser at these same frequencies, which is not fully suppressed by the $f_{o}$ servo. Fig. 8(b) is an expanded view around the carrier, where we see the PZT resonance at $\sim 22 \mathrm{kHz}$ and some additional noise of acoustic origin at frequencies less than a few kilohertz. With larger servo bandwidth and improved environmental isolation of the laser, we should be able to further suppress these features. Nonetheless, in the current state a distinct carrier with good SNR is present, as seen in Fig. 8(c). For this trace, an additional mixing with a synthesizer was employed to shift $f_{b}$ to $1 \mathrm{kHz}$, which is within the range of the Fourier-transform spectrum analyzer. The recorded linewidth is measurement-limited at about $15 \mathrm{mHz}$. While they are not fully orthogonal in their control, we see no evidence that
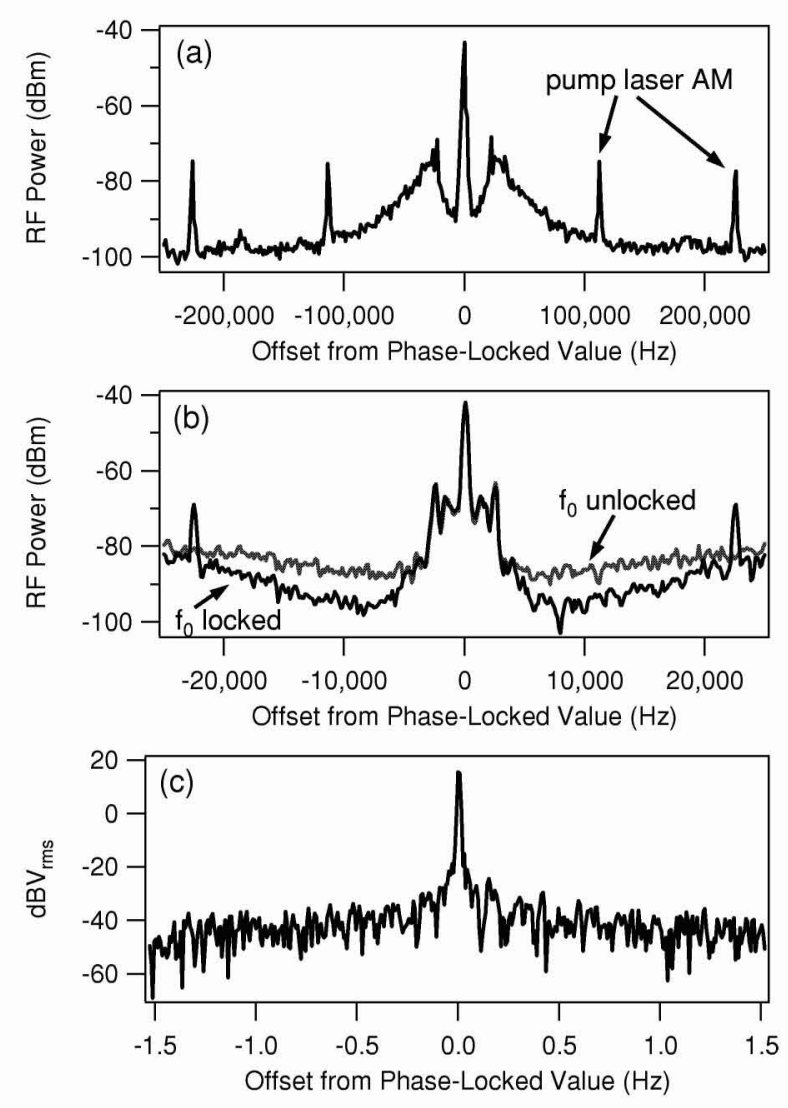

Fig. 8. Frequency spectrum of phase-locked beat $f_{b}$ with the $\mathrm{Hg}^{+}$standard. (a) RF spectrum analyzer measurement with resolution bandwidth of $1 \mathrm{kHz}$. (b) RF spectrum analyzer measurement with resolution bandwidth of $300 \mathrm{~Hz}$. (c) Measurement with a Fourier-transform spectrum analyzer having effective resolution of $15 \mathrm{mHz}$.

the two phase-lock loops have a deleterious effect on each other. To the contrary, we see a reduction in noise in the $f_{b}$ phase lock when $f_{o}$ is also phase locked [see Fig. 8(b)]. Others have previously reported tight phase locking of $f_{o}$ [31], [32], but to our knowledge, this is the first evidence that the control of both degrees of freedom of the octave-spanning comb can be at a level significantly less than $1 \mathrm{~Hz}$, relative to an optical standard.

With $f_{o}$ and $f_{b}$ thus stabilized to the $\mathrm{Hg}^{+}$standard, we can make a first check of the linewidth of the other comb elements by heterodyning a second comb tooth against the $657-\mathrm{nm}$ light from the Ca standard. The spectrum of this beat, as measured with an RF spectrum analyzer, is plotted as the dashed line in Fig. 9(a), with the same data plotted on an expanded frequency scale shown in the inset. One factor contributing to the frequency width of this heterodyne signal is the phase noise (Doppler broadening) introduced by mechanical and thermal fluctuations in the $180-\mathrm{m}$ single-mode fiber that delivers the $563-\mathrm{nm}$ laser light to the femtosecond laser. The 563-nm light is spectrally broadened and the servo controlling $f_{b}$ then transfers the same noise to the femtosecond comb. To eliminate this effect, an active noise cancellation servo has been implemented for the 180-m fiber [33], [34], and in-loop data from this servo indicate that the subhertz linewidth of the $\mathrm{Hg}^{+}$standard can be delivered over fiber. The same effective heterodyne signal between the $\mathrm{Hg}^{+}$and $\mathrm{Ca}$ standards with the Doppler cancellation 

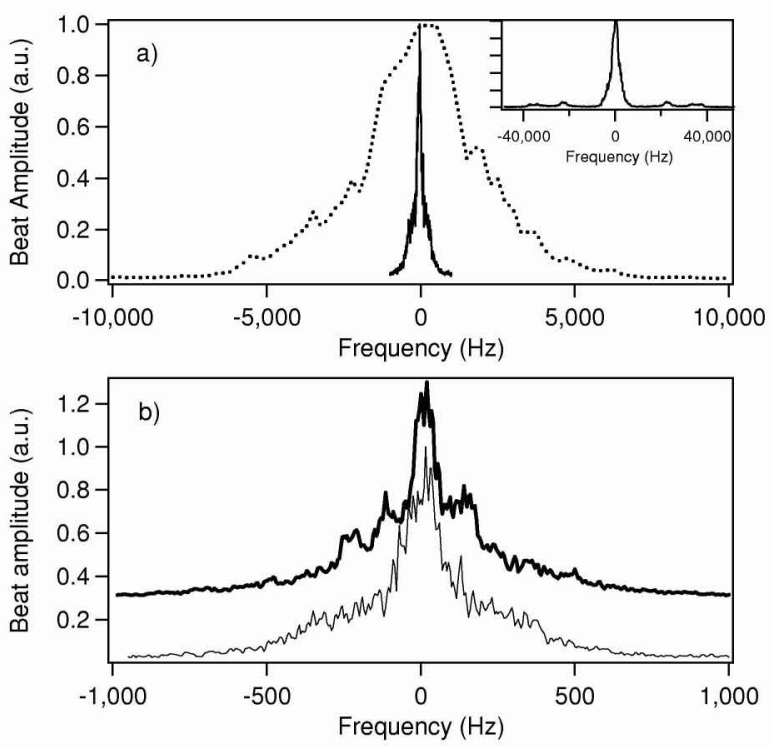

Fig. 9. (a) Effective heterodyne of $\mathrm{Hg}^{+}$and $\mathrm{Ca}$ optical standards via a femtosecond comb that is frequency-stabilized to the $\mathrm{Hg}^{+}$standard. Two traces were recorded when the Doppler cancellation for the $\mathrm{Hg}^{+}$delivery fiber was OFF (dotted line) and ON (solid line). Inset shows the heterodyne with no Doppler cancellation at an expanded scale. (b) Lower trace is an expanded view of the solid line of plot (a). Linewidth is comparable to the measured spectral broadening due to two trips of the Ca-stabilized light through $10 \mathrm{~m}$ of optical fiber, which is shown in the upper trace. $10-\mathrm{Hz}$ resolution bandwidth was used for these measurements.

servo closed is plotted as the solid line in Fig. 9(a). As seen, the noise cancellation has a dramatic effect, decreasing the measured linewidth by a factor of $\sim 25$, down to about $150 \mathrm{~Hz}$.

This $150-\mathrm{Hz}$ linewidth provides proof that the modes of the femtosecond laser comb can track the $\mathrm{Hg}^{+}$standard at this same level. However, it is likely that the modes of the comb are even narrower, as both the $10 \mathrm{~m}$ of optical fiber that transports the $\mathrm{Ca}$ light to the femtosecond laser and the linewidth of the Ca-stabilized $657-\mathrm{nm}$ diode laser also contribute to the measured $150-\mathrm{Hz}$ linewidth. Independent measurements place a generous upper limit of $\sim 100 \mathrm{~Hz}$ on the diode laser linewidth. The 10-m optical fiber also introduces broadening at about this same level as shown in Fig. 9(b). To measure the fiber-induced broadening, stabilized light from the $\mathrm{Ca}$ experiment was sent through the 10-m fiber, frequency-shifted by an AOM, and returned through the same fiber to beat against the source. This heterodyne is displayed as the upper trace in Fig. 9(b). For comparison, the lower trace again shows the $150-\mathrm{Hz}$ linewidth of the $\mathrm{Ca}-\mathrm{Hg}^{+}$ effective beat described above [i.e., the solid trace of Fig. 9(a)]. The linewidths are comparable. Although the fiber noise results from two trips through the $10-\mathrm{m}$ fiber, the linewidth due to a single pass is still expected to be well above $75 \mathrm{~Hz}$. It is clear that Doppler cancellation is also required on even the relatively short $10-\mathrm{m}$ fiber link if we are to resolve the true linewidth of the effective beat between the $\mathrm{Hg}^{+}$and Ca standards. More rigorous tests of the femtosecond comb are also required. While the spectra of Figs. 7-9 offer evidence that the comb teeth are narrow, this can be verified unambiguously by heterodyning two independently stabilized frequency combs and measuring these linewidths across the broad optical spectrum. Such measurements are in progress.

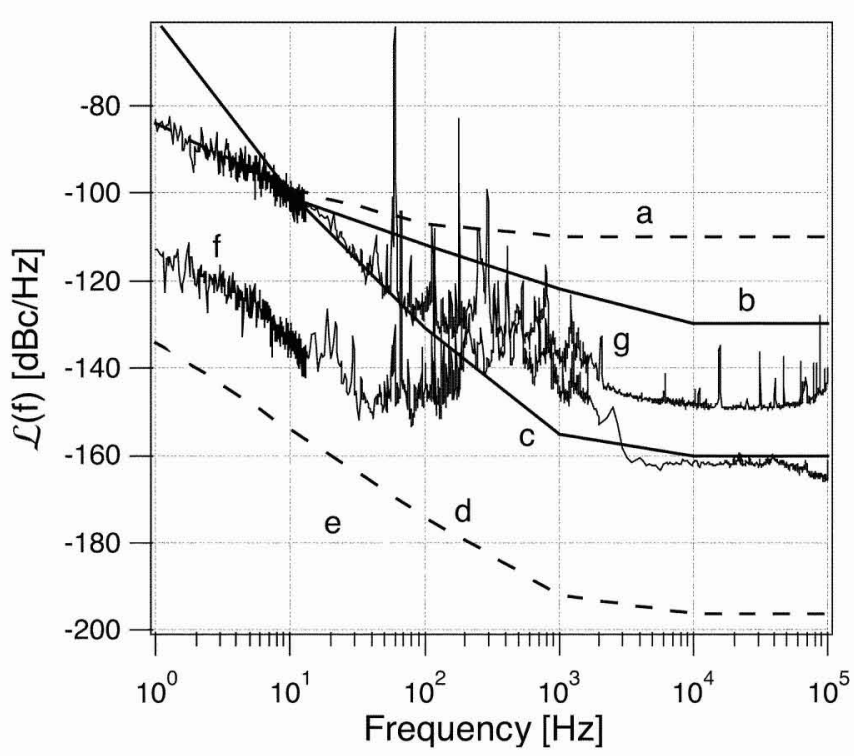

Fig. 10. Comparison of the phase noise spectrum $L(f)$ of various precision oscillators and synthesizers at $1 \mathrm{GHz}$. Where necessary, multiplication or division of the phase noise from the usual carrier frequency for each device has been accounted for: (a) low-noise quartz oscillator; (b) low-noise RF/microwave synthesizer; (c) sapphire loaded cavity oscillator; (d) projected phase noise of $\mathrm{Ca}$ optical standard with Allan deviation of $6 \times 10^{-17} \tau^{-1}+2 \times 10^{-16} \tau^{-1 / 2}$; (e) projected phase noise of visible laser that serves as reference for the $\mathrm{Hg}^{+}$optical standard based on the measured Allan deviation of $1.5 \times 10^{-16} \tau^{-1}+4 \times 10^{-16}$; (f) optical pulse train output of femtosecond laser synthesizer; and (g) microwave electronic output of femtosecond laser synthesizer

\section{B. Generation of Low-Noise Microwave Signals}

It is of interest to also know how well the femtosecond comb transfers the properties of the optical standards into the microwave regime (i.e., to $f_{r}$ ) and what potential exists if we are successful in this endeavor. As a point of reference, Fig. 10 displays the single-side-band phase-noise spectrum $\mathcal{L}(f)$ of several oscillators, frequency standards, and synthesizers from both the optical and microwave domains. In this plot, comparison is made at a 1-GHz carrier frequency, so where necessary multiplication or division of the phase noise from the usual carrier frequency for each device has been accounted for. Curves (a)-(c) show typical values of $\mathcal{L}(f)$ for a high-quality quartz crystal oscillator, a low-noise commercial RF/microwave synthesizer, and a sapphire-loaded cavity oscillator (SLOC). This type of sapphire oscillator is recognized as one of the lowest noise microwave sources that exist. For comparison, curves (d) and (e) show the projected phase noise of the Ca optical standard as well as the cavity-stabilized laser oscillator for the $\mathrm{Hg}^{+}$standard. If their properties can be successfully transferred to $1 \mathrm{GHz}$, these optical sources potentially offer phase noise across the spectrum lower than that of the best microwave sources, and a potential decrease below $1 \mathrm{kHz}$ of 30 to $60 \mathrm{~dB}$. Clearly, there is a large potential payoff in the phase noise levels offered by optical standards and oscillators compared to the best existing microwave options.

An important question, then, is how well the femtosecond frequency comb is able to transfer the excellent noise properties of these optical oscillators to the microwave domain. Curves (f) and (g) of Fig. 10 show the current level of performance at 
$1 \mathrm{GHz}$. Curve (f) is the measured residual phase noise of the femtosecond synthesizer when one examines the optical pulse train output. Curve $(\mathrm{g})$ is the measured residual phase noise of the femtosecond synthesizer when one examines the microwave (electronic) output. These data were obtained by phase locking the combs of two different mode-locked lasers to a common stabilized laser diode and comparing their emerging pulse trains. In one case, (f), an optical nonlinear cross-correlation measurement, was employed and in the other case, (g), the two pulse trains, was photodetected to provide 1-GHz signals that are subsequently compared in an electronic mixer. Further experimental details are given in [35]. It is worth noting that the contribution of the phase-locking system to the noise at $f_{r}$ from the femtosecond laser is still well below that of curves (f) and $(\mathrm{g})$. For the case of the $\int_{0}$ lock, this can be determined by subtracting approximately $110 \mathrm{~dB}$ from the trace of Fig. 7 .

Comparison of curves ( $\mathrm{f}$ ) and ( $\mathrm{g}$ ) to the others shows the encouraging results that are already obtained at this early stage as well as the potential for continued improvement. The residual phase noise of the optical pulse train output of the femtosecond laser synthesizer is already at a level that begins to approach the projections of the optical standards themselves, at least for frequencies below $100 \mathrm{~Hz}$. Corresponding time-domain measurements demonstrated an instability of the femtosecond laserbased synthesizer of $\sim 2 \times 10^{-15} \tau^{-1}$ ( $\tau$ measured in seconds) [35], which is consistent with instability calculated from the integration of curve (f). The excess noise in the range from 0.1 to $1 \mathrm{kHz}$ is attributed to undamped mechanical vibrations in the laser and the measurement system that do not pose a fundamental limitation, and the high-frequency noise floor is believed to be that of the measurement system.

Of greater significance is the $\sim 30 \mathrm{~dB}$ increase in phase noise that arises in the photodetection process when the optical pulse trains are converted to electronic signals [curve (g)]. As discussed in more detail by Ivanov et al. [36], amplitude-to-phase noise conversion and pointing instability may be sources of this excess noise. However, spatial filtering in optical filters and active stabilization of the optical power reaching the detectors still has not yet allowed us to reduce the the level of phase noise to that of curve (f). Clearly, this is an interesting and important problem that needs to be understood if the full potential of the femtosecond-laser-based synthesizer is to be realized. However, it is significant that even in the present situation, the residual phase noise above $10 \mathrm{~Hz}$ on the microwave output of the femtosecond-laser-based synthesizer is better than that of high-quality crystal quartz and microwave synthesizers. Indeed, time-domain measurements and integration of curve (g) verify that the microwave output of the femtosecond synthesizer can support instabilities of $\sim 2 \times 10^{-11} \tau^{-1}$, which is a level attained by only a few microwave sources.

\section{CONCLUSION}

In this paper, we have provided a description of some state-of-the-art femtosecond laser tools and have offered our perspective on where and how they can be of value in the rapidly evolving field of optical frequency metrology. With a high-repetition-rate femtosecond laser that directly emits a very broad spectrum, we have demonstrated a robust clockwork that will ultimately allow optical clocks to operate for near-indefinite periods. We have also demonstrated that the linewidth of a very narrow optical oscillator can be transferred to the elements of a femtosecond comb at a measurement-limited level of $<150 \mathrm{~Hz}$. Measurements of the phase-lock loops controlling the femtosecond comb indicate that the linewidth of the comb teeth could actually be less than $1 \mathrm{~Hz}$. Finally, we have shown that the femtosecond laser can function as a low-noise synthesizer in transferring the properties of optical standards to a microwave frequency of $1 \mathrm{GHz}$. While there is still much progress to be made in this area, we believe that optical frequency standards combined with femtosecond lasers will be legitimate low-noise microwave sources, challenging and surpassing the performance of the best current microwave standards.

\section{ACKNOWLEDGMENT}

The authors would like to thank R. Windeler of OFS Laboratories for generously providing the microstructure optical fiber. They also wish to thank $\mathrm{E}$. Ivanov for his many stimulating discussions and assistance with microwave measurements.

\section{REFERENCES}

[1] T. Udem, J. Reichert, R. Holzwarth, and T. W. Hänsch, "Absolute optical frequency measurement of the cesium $D_{1}$ line with a mode-locked laser," Phys. Rev. Lett., vol. 82, pp. 3568-3571, 1999.

[2] - "Accurate measurement of large optical frequency differences with a mode-locked laser," Opt. Lett., vol. 24, pp. 881-883, 1999.

[3] J. L. Hall, J. Ye, S. A. Diddams, L.-S. Ma, S. T. Cundiff, and D. J. Jones, "Ultrasensitive spectroscopy, the ultrastable lasers, the ultrafast lasers, and the seriously nonlinear fiber: A new alliance for physics and metrology," IEEE J. Quantum Electron., vol. 37, pp. 1482-1492, 2001.

[4] L. Hollberg, C. W. Oates, E. A. Curtis, E. N. Ivanov, S. A. Diddams, T. Udem, H. G. Robinson, J. C. Bergquist, R. J. Rafac, W. M. Itano, R. E. Drullinger, and D. J. Wineland, "Optical frequency standards and measurements," IEEE J. Quantum Electon., vol. 37, pp. 1502-1513, 2001.

[5] T. Udem, R. Holzwarth, and T. W. Hänsch, "Optical frequency metrology," Nature, vol. 416, pp. 233-237, 2002.

[6] S. A. Diddams, T. Udem, J. C. Bergquist, E. A. Curtis, R. E. Drullinger, L. Hollberg, W. M. Itano, W. D. Lee, C. W. Oates, K. R. Vogel, and D. J. Wineland, "An optical clock based on a single trapped ${ }^{199} \mathrm{Hg}^{+}$ion," Science, vol. 293, pp. 825-828, 2001.

[7] J. Ye, L.-S. Ma, and J. L. Hall, "Molecular iodine clock," Phys. Rev. Lett., vol. 87, pp. 270 801/1-4, 2001.

[8] S. A. Diddams, L. Hollberg, L.-S. Ma, and L. Robertsson, "A femtosecond-laser-based optical clockwork with instability $\leq 6.3 \times 10^{-16}$ in $1 \mathrm{~s}, "$ Opt. Lett., vol. 27, pp. 58-60, 2002.

[9] R. Holzwarth, T. Udem, T. W. Hänsch, J. C. Knight, W. J. Wadsworth, and P. S. J. Russell, "Optical frequency synthesizer for precision spectroscopy," Phys. Rev. Lett., vol. 85, pp. 2264-2267, 2000.

[10] R. Holzwarth, "Measuring the frequency of light using femtosecond laser pulses," Ph.D. dissertation, Ludwig-Maximilians-Universität, München, Germany, 2001.

[11] J. Stenger, H. Schnatz, C. Tamm, and H. R. Telle, "Ultraprecise measurement of optical frequency ratios," Phys. Rev. Lett., vol. 88, pp. $073601-1-4,2002$.

[12] D. J. Jones, S. A. Diddams, J. K. Ranka, A. Stentz, R. S. Windeler, J. L Hall, and S. T. Cundiff, "Carrier-envelope phase control of femtosecond modelocked lasers and direct optical frequency synthesis," Science, vol. 288, pp. 635-639, 2000.

[13] J. K. Ranka, R. S. Windeler, and A. J. Stentz, "Visible continuum generation in air silica microstructure optical fibers with anomalous dispersion at $800 \mathrm{~nm}$," Opt. Lett., vol. 25, pp. 25-27, 2000.

[14] K. L. Corwin, N. R. Newbury, J. M. Dudley, S. Coen, S. A. Diddams, K. Weber, and R. S. Windeler, "Fundamental noise limitations to supercontinuum generation in microstructure fiber," Phys. Rev. Lett., 2003, to be published. 
[15] R. Ell, U. Morgner, F.X. Kärtner, J. G. Fujimoto, E. P. Ippen, V. Scheuer, G. Angelow, T. Tschudi, M. J. Lederer, A. Boiko, and B. Luther-Davies, "Generation of 5-fs pulses and octave-spanning spectra directly from a ti:sapphire laser," Opt. Lett., vol. 26, pp. 373-375, 2001.

[16] U. Morgner, R. Ell, G. Metzler, T. R. Schibli, F. X. Kärtner, J. G. Fujimoto, H. A. Haus, and E. P. Ippen, "Nonlinear optics with phase-controlled pulses in the sub-two-cycle regime," Phys. Rev. Lett., vol. 86, pp. $5462-5465,2001$.

[17] A. Bartels, T. Dekorsy, and H. Kurz, "Femtosecond ti:sapphire ring laser with a 2-GHz repetition rate and its application in time-resolved spectroscopy," Opt. Lett., vol. 24, pp. 996-998, 1999.

[18] A. Bartels and H. Kurz, "Generation of a broadband continuum by a ti:sapphire femtosecond oscillator with a 1-GHz repetition rate," Opt. Lett., vol. 27, pp. 1839-1841, 2002.

[19] T. M. Ramond, S. A. Diddams, L. Hollberg, and A. Bartels, "Phase coherent link from optical to microwave frequencies via the broadband continuum from a 1 GHZ ti:Sapphire femtosecond oscillator," Opt. Lett., vol. 27, pp. 1842-1844, 2002.

[20] C. W. Oates, F. Bondu, R. W. Fox, and L. Hollberg, "A diode-laser optical frequency standard based on laser-cooled ca atoms: Sub-kilohertz spectroscopy by optical shelving detection," Eur. Phys. J. D, vol. 7, pp. 449-460, 1999.

[21] C. W. Oates, E. A. Curtis, and L. Hollberg, "Improved short-term stability of optical frequency standards: approaching $1 \mathrm{hz}$ in $1 \mathrm{~s}$ with the ca standard at 657 nm," Opt. Lett., vol. 25, pp. 1603-1605, 2000.

[22] J. L. Hall, "Optical frequency measurement: 40 years of technology revolutions," IEEE J. Select. Topics Quantum Electron., vol. 6, pp. $1136-1144,2000$.

[23] B. C. Young, F. C. Cruz, W. M. Itano, and J. C. Bergquist, "Visible lasers with subhertz linewidths," Phys. Rev. Lett., vol. 82, pp. 3799-3802, 1999.

[24] H. R. Telle, B. Lipphardt, and J. Stenger, "Kerr-lens, mode-locked lasers as transfer oscillators for optical frequency measurements," App. Phys. $B$, vol. 74, pp. 1-6, 2002

[25] J. Stenger, H. Schnatz, C. Tamm, and H. R. Telle, "Ultraprecise measurement of optical frequency ratios," Phys. Rev. Lett., vol. 88, pp. $073601 / 1-4,2002$.

[26] T. H. Yoon, A. Marian, J. L. Hall, and J. Ye, "Phase-coherent multilevel two-photon transitions in cold rb atoms: Ultrahigh-resolution spectroscopy via frequency-stabilized femtosecond laser," Phys. Rev. A, vol. 63, pp. $011402(\mathrm{R}) / 1-4,2001$.

[27] S. Schiller, "Spectrometry with frequency combs," Opt. Lett., vol. 27, pp. $766-768,2002$

[28] R. J. Rafac, B. C. Young, J. A. Beall, W. M. Itano, D. J. Wineland, and J. C. Bergquist, "Sub-dekahertz ultraviolet spectroscopy of ${ }^{199} \mathrm{Hg}_{\mathrm{g}}{ }^{+}$," Phys. Rev. Lett., vol. 85, pp. 2462-2465, 2000.

[29] S. A. Diddams, T. Udem, K. R. Vogel, C. W. Oates, E. A. Curtis, R. S. Windeler, A. Bartels, J. C. Bergquist, and L. Hollberg, "Laser frequency stabilization, standards, measurement, and applications," in Proc. SPIE, J. L. Hall and J. Ye, Eds., 2001.

[30] T. Udem, S. A. Diddams, K. R. Vogel, C. W. Oates, E. A. Curtis, W. D. Lee, W.M. Itano, R. E. Drulling er, J. C. Bergquist, andL. Hollberg, "Absolute frequency measurements of the $\mathrm{Hg}^{+}$and ca optical clock transitionswith a femtosecond laser," Phys. Rev. Lett., vol. 86, pp.4499-4996, 2001.

[31] T. M. Fortier, D. J. Jones, J. Ye, S. T. Cundiff, and R. S. Windeler, "Long-term carrier-envelope phase coherence," Opt. Lett., vol. 27, pp. $1436-1438,2002$

[32] F. W. Helbing, G. Steinmeyer, J. Stenger, H. R. Telle, and U. Keller, "Carrier-envelope-offset dynamics and stabilization of femtosecond pulses," Appl. Phys. B, vol. 74, pp. S35-S42, 2002.

[33] L.-S. Ma, P. Jungner, J. Ye, and J. L. Hall, "Delivering the same optical frequency at two places: Accurate cancellation of phase noise introduced by an optical fiber or other time-varying path," Opt. Lett., vol. 19, pp. $1777-1779,1994$

[34] B. C. Young, R. J. Rafac, J. A. Beall, F. C. Cruz, W. M. Itano, D. J. Wineland, and J. C. Bergquist, "Hg ${ }^{+}$optical frequency standard: Recent progress," in Laser Spectroscopy, XIV, R. Blatt, J. Eschner, D. Leibfried, and F. Schmidt-Kaler, Eds., Singapore, 1999.

[35] A. Bartels, S. A. Diddams, T. M. Ramond, and L. Hollberg, "Modelocked laser pulse trains with subfemtosecond timing jitter synchronized to an optical reference oscillator," Opt. Lett., to be published.

[36] E. N. Ivanov, L. Hollberg, and S. A. Diddams, "Analysis of noise mechanisms limiting the frequency stability of microwave signals generated with a femtosecond laser," IEEE J. Select. Topics Ouantum Electron., vol. 9, pp. XXX-XXX, July/Aug. 2003.
Scott A. Diddams was born in Gallup, NM, in 1967. He received the B.A. degree in physics from Bethel College, St. Paul, MN, in 1989 and the Ph.D. degree in optical science from the University of New Mexico, Albuquerque, in 1996.

Between 1996 and 2000, he did postdoctoral work at JLA (a joint institute of the National Institute of Standards and Technology and the University of Colorado), where he was supported in part by a National Research Council fellowship. Currently, he works as a Staff Physicist in the Time and Frequency Division, National Institute of Standards and Technology (NIST), Boulder, CO, where his research interests include the fields of nonlinear optics, ultrafast lasers and phenomena, and precision spectroscopy and metrology.

Albrecht Bartels was born in 1972 in Orsoy, Germany. He received the diploma in physics from RWTH Aachen, Germany, in 1997.

In his graduate work he dealt with time-resolved studies of coherent excitations in solid materials and built the first Ti:sapphire femtosecond laser with repetition rates above $1 \mathrm{GHz}$. After his graduation from the Institut für Halbleitertechnik, RWTH Aachen, in 2000, he led GigaOptics GmbH while at the same time having postdoc positions at RWTH Aachen and Bonn University. He joined the National Institute of Standards and Technology, Boulder, CO, in 2002 to explore new possibilities with gigahertz femtosecond lasers for the synthesis of optical and microwave frequencies.

Tanya M. Ramond was born in 1972 in New Haven, CT. She received the B.A. degree in physics from Bryn Mawr College, Bryn Mawr, PA, and the Ph.D. degree in physics from the University of Colorado, Boulder, 2001.

She is currently a National Research Council postdoctoral Fellow at the National Institute of Standards and Technology (NIST), Boulder, CO. Her research interests include the application of femtosecond lasers for frequency metrology.

Chris W. Oates was born in Glendale, CA, on April 12, 1962. He received the B.S. degree in physics from Stanford University, Stanford, CA, in 1984 and the Ph.D. degree in physics from the University of Colorado, Boulder, in 1995. His thesis work was performed in the laboratory of J. L. Hall

He spent two years teaching with Peace Corps. Later, he joined the National Institute of Standards and Technology (NIST), Boulder, CO, as an NRC Postdoctoral Fellow and began work on an optical atomic clock based on lasercooled Ca atoms. He became a NIST Staff Physicist in 1998 and has continued his work on laser cooling, optical frequency standards, and the frequency stabilization of semiconductor lasers

S. Bize, photograph and biography not available at the time of publication.

E. A. Curtis was born in Buffalo, New York, in 1976. She received the B.S. degree in physics from Duke University in 1998 and the Ph.D. degree from the University of Colorado, Boulder, in 2003.

She currently holds a Royal Society research fellowship and is a Postdoctoral Researcher at Imperial College, London, U.K., working towards Bose-Einstein condensation on magnetic-media-based "atom chips."

J. C. Bergquist, photograph and biography not available at the time of publication.

Leo Hollberg (A'89) was born in Denver, CO, in 1952. He received the B.S. degree in physics from Stanford University, Stanford, CA, in 1976. He received the Ph.D. degree in physics from the University of Colorado, Boulder, in 1984, for research in high-resolution laser spectroscopy done with J. Hall at JLA.

He was a Postdoctoral Researcher in 1984 and 1985 at AT\&T Bell Laboratories working with S. Chu on laser cooling and with R. Slusher on squeezed states. Since then, has been at the Time and Frequency Division, National Institute of Standards and Technology (NIST), Boulder, CO, doing research on high-resolution spectroscopy of laser-cooled and trapped atoms, the development of semiconductor lasers for scientific and technical applications, optical coherence effects of driven multilevel atoms, and optical frequency standards and measurements. 